\title{
F-PURE THRESHOLDS AND F-JUMPING EXPONENTS IN DIMENSION TWO
}

\author{
NOBUO HARA \\ with an Appendix by Paul Monsky
}

\begin{abstract}
Using an argument based on an idea of Monsky, we prove the rationality of the F-pure thresholds of curve singularities on a smooth surface defined over a finite field. More generally, we prove in this setting the rationality and discreteness of F-jumping exponents, the smallest positive one of which is the F-pure threshold. We also give a lower bound for F-pure thresholds in the homogeneous case.
\end{abstract}

\section{Introduction}

Let $D$ be an effective divisor on a smooth variety defined over a perfect field of characteristic $p>0$. The F-pure threshold of the pair $(X, D)$ at $x \in X$ is defined to be the real number

$$
\operatorname{fpt}_{x}(X, D)=\sup \left\{t \in \mathbb{R}_{\geq 0} \mid(X, t D) \text { is F-pure at } x\right\},
$$

where the pair $(X, t D)$ is said to be $\mathrm{F}$-pure at $x \in X$ if the natural inclusion map $\mathcal{O}_{X, x} \hookrightarrow \mathcal{O}_{X}(t(q-1) D)_{x}^{1 / q}$ splits as an $\mathcal{O}_{X, x}$-module homomorphism for all powers $q=p^{e}$ of $p$. F-purity of pairs is introduced in [HW2] as a characteristic $p$ analog of the notion of $\log$ canonical singularity of pairs. Indeed F-purity enjoys several local properties similar to log canonical singularity, although the definition is very different. Thus we are led to the concept of F-pure threshold as an "F-pure version" of the $\log$ canonical threshold studied in [K, Section 8]. However, it is not a priori clear that F-pure thresholds are rational numbers, while log canonical thresholds are clearly rational by definition. Using Paul Monsky's idea, we shall give an affirmative answer to the simplest non-trivial case of this question.

Theorem 1.1. Let $R=\mathbb{F}_{q}[[x, y]]$ be a two-dimensional complete regular local ring over a finite field $\mathbb{F}_{q}$ and let $D=\operatorname{div}_{X}(f)$ be the divisor on $X=\operatorname{Spec} R$ defined by a nonzero element $f \in R$. Then the F-pure threshold $\operatorname{fpt}(X, D)$ is a rational number.

The crucial point of the proof of this theorem is that the function $\varphi_{f}(t)$ defined by $\varphi_{f}(a / q)=q^{-2} \ell\left(R /\left(x^{q}, y^{q}, f^{a}\right)\right)$ for a power $q$ of $p$ and $0 \leq a<q$, is a $p$-fractal as is

Received by the editors February 15, 2006.

This work grew from a conversation with Paul Monsky when the author participated in the "BIRS Workshop on Commutative Algebra: Homological and Birational Theory" held September 11-16, 2004. The author thanks Monsky for his idea and careful comments, and the organizers of the workshop and BIRS for giving them such an opportunity. He also thanks the referee for invaluable comments. The author is partially supported by Grant-in-Aid for Scientific Reserach, Japan. 
defined and proved in [MT]. Roughly, this implies that the graph of $\varphi_{f}$ has a sort of self-similarity under $q$-times magnification near the $\mathrm{F}$-pure threshold $c=\operatorname{fpt}(X, D)=$ $\inf \left\{t \in[0,1] \mid \varphi_{f}(t)=1\right\}$. Accordingly, the $p$-adic expansion of $c$ becomes eventually periodic, from which the rationality follows.

We can extend our method to prove the rationality and discreteness of $F$-jumping exponents of a pair $(X, D)$, where $X=\operatorname{Spec} R$ and $D=\operatorname{div}_{X}(f)$ are as in Theorem 1.1. Given any nonnegative real number $t$, the generalized test ideal $\tau\left(f^{t}\right)=\tau(X, t D)$ of the pair $(R, f)$ (or, of the pair $(X, D))$ is defined via $f^{t}$-tight closure [HY]. This ideal satisfies the property that $\tau\left(f^{t}\right) \supseteq \tau\left(f^{t^{\prime}}\right)$ if $t \leq t^{\prime}$. We say that a positive real number $c$ is an F-jumping exponent of $(R, f)$ if it is the infimum of those $t \in \mathbb{R}_{\geq 0}$ for which $\tau\left(f^{t}\right)=\tau\left(f^{c}\right)$. This may be considered a characteristic $p$ analog of the notion of jumping coefficients for multiplier ideals; see [ELSV]. It is easily seen that jumping coefficients for multiplier ideals of a fixed pair $(X, D)$ form a discrete set of rational numbers. The discreteness of the set of jumping coefficients is used to prove a uniform Artin-Rees theorem in [ELSV], and it is natural to ask if these properties also hold true for F-jumping exponents. We answer this question affirmatively in Theorem 2.17, under the assumptions of Theorem 1.1, using the results of Monsky's appendix. Indeed the arguments show the rationality and discreteness of "F-thresholds" attached to $f$ in the sense of $[\mathrm{MTW}]$.

Given any non-zero $f \in k[[x, y]]$ with char $k=p>0$, it is not easy to compute the F-pure threshold even if we know that it is rational. In Section 4 of this paper, we give further results about the F-pure threshold and the function $\varphi_{f}(t)$ when $f$ is a homogeneous polynomial of degree $d$. Namely, we give a lower bound for the F-pure threshold and a list of its possible values in low degrees. Also, we show that $\varphi_{f}(t)$

differs from a quadratic function by at most $c / p^{2}$, where both the quadratic function and $c$ depend only on $d$. In his paper [M3], Monsky obtained results overlapping these by using Mason's theorem, while we use more geometric tools developed in [HW1].

This paper is organized as follows. In Section 2, we give results on F-singularities of pairs, generalized test ideals and F-jumping exponents. These results combine with results from the appendix by Monsky to yield Theorem 2.17. Section 3 is devoted to studying the homogeneous case.

\section{F-singularities of pairs, generalized test ideals, and F-jumping exponents}

In this section, we review the theory of F-singularities of pairs [HW2] and generalized test ideals [HY]. Although our theory has been developed for a much wider class of rings of characteristic $p$, we will restrict ourselves to following situation.

Notation 2.1. Let $R=k\left[\left[x_{1}, \ldots, x_{d}\right]\right]$ denote a $d$-dimensional complete regular local ring over a perfect field $k$ with the maximal ideal $\mathfrak{m}=\left(x_{1}, \ldots, x_{d}\right)$. We assume that char $k=p>0$ unless otherwise specified, and use the letter $q$ to denote a power $p^{e}$ of $p$. The $q$ th Frobenius power $I^{[q]}$ of an ideal $I$ of $R$ is the ideal generated by the $q$ th powers of elements of $I$.

Let $0 \neq f \in \mathfrak{m}$ and let $t$ be a nonnegative real number. Consider the pair $(X, t D)$ where $X=\operatorname{Spec} R$ and $D=\operatorname{div}_{X}(f)$. Write the $\mathbb{R}$-divisor $t D$ as a linear combination $\sum_{i=1}^{r} t_{i} D_{i}$ of prime divisors $D_{i}$ with coefficients $t_{i} \in \mathbb{R}$, and let $\lfloor t D\rfloor$ be obtained from 
$t D$ by replacing each $t_{i}$ by its round-down $\left\lfloor t_{i}\right\rfloor$. Similarly, $\lceil t D\rceil$ is obtained from $t D$ by replacing each $t_{i}$ by its round-up $\left\lceil t_{i}\right\rceil$. We denote by $\mathcal{O}_{X}(t D)=\mathcal{O}_{X}(\lfloor t D\rfloor)$ the sheaf associated to the integral divisor $\lfloor t D\rfloor$.

We often speak of the pair $\left(R, f^{t}\right)$ instead of the pair $(X, t D)$, and we use these two expressions interchangeably.

In characteristic $p>0$, we denote the absolute Frobenius morphism of $X$ and the associated ring homomorphism by $F: X \rightarrow X$ and $F: \mathcal{O}_{X} \rightarrow F_{*} \mathcal{O}_{X}$, respectively.

Definition 2.2 ([HW2]). Let the notation be as above.

(1) We say that the pair $(X, t D)$ is $F$-pure if the composition map

$$
F^{e}: \mathcal{O}_{X} \rightarrow F_{*}^{e} \mathcal{O}_{X}((q-1) t D),
$$

of the eth Frobenius map $\mathcal{O}_{X} \rightarrow F_{*}^{e} \mathcal{O}_{X}$ and the natural inclusion $F_{*}^{e} \mathcal{O}_{X} \hookrightarrow$ $F_{*}^{e} \mathcal{O}_{X}((q-1) t D)$, splits as an $\mathcal{O}_{X}$-module homomorphism for every $q=p^{e}$.

(2) The pair $(X, t D)$ is strongly F-regular if for every effective divisor $E$ on $X$, there exists $q=p^{e}$ such that the map $F^{e}: \mathcal{O}_{X} \rightarrow F_{*}^{e} \mathcal{O}_{X}((q-1) t D+E)$ splits as an $\mathcal{O}_{X}$-module homomorphism. Equivalently, ${ }^{1}(X, t D)$ is strongly F-regular if the map $F^{e}: \mathcal{O}_{X} \rightarrow F_{*}^{e} \mathcal{O}_{X}(\lceil q t D\rceil)$ splits for all sufficiently large $q=p^{e}$.

Proposition 2.3 ([HW2, Prpoposition 2.2]).

(1) If $(X, t D)$ is strongly F-regular, then $(X, t D)$ is F-pure.

(2) If $(X, t D)$ is $F$-pure, then $\lceil t D\rceil$ is reduced (i.e., $t_{i} \leq 1$ for every $\left.i\right)$.

(3) If $(X, t D)$ is strongly $F$-regular, then $\lfloor t D\rfloor=0$ (i.e., $t_{i}<1$ for every $i$ ).

(4) If $(X, t D)$ is F-pure (resp. strongly F-regular), then so is $\left(X, t^{\prime} D\right)$ for every $t^{\prime} \in \mathbb{R}$ with $0 \leq t^{\prime} \leq t$

Since $(X, 0)$ is strongly F-regular (and so F-pure) under our assumption that $R$ is regular, the following "F-analog" of the log canonical threshold is defined; cf. $[\mathrm{K}]$.

Definition 2.4. Let $X=\operatorname{Spec} R$ and $D=\operatorname{div}_{X}(f)$ be as in 2.1. We define the F-pure threshold of the pair $(X, D)$ by

$$
\operatorname{fpt}(X, D)=\sup \left\{t \in \mathbb{R}_{\geq 0} \mid(X, t D) \text { is F-pure }\right\} .
$$

We note that $0<\operatorname{fpt}(X, D) \leq 1$ if $D \neq 0$ and that $\operatorname{fpt}(X, 0)=\infty$.

Remark 2.5. It easily follows from the definition (and its rephrased form in 2.2(2)) that $(X, t D)$ is strongly F-regular if and only if $t<\operatorname{fpt}(X, D)$. In other words, strong F-regularity of pairs is an open condition with respect to the coefficient, and

$$
\operatorname{fpt}(X, D)=\sup \left\{t \in \mathbb{R}_{\geq 0} \mid(X, t D) \text { is strongly F-regular }\right\}
$$

cf. Proposition 2.8 (4). On the other hand, as we will see in Proposition 2.6 below, F-purity of pairs is a closed condition with respect to the coefficient, i.e., $(X, t D)$ is F-pure if and only if $t \leq \operatorname{fpt}(X, D)$.

Proposition 2.6. Let $(X, D)$ be as in 2.4, and suppose $c=\operatorname{fpt}(X, D)<\infty$. For each $e \in \mathbb{N}$, let $r_{e} \in \mathbb{N}$ be the integer such that the map $F^{e}: \mathcal{O}_{X} \rightarrow F_{*}^{e} \mathcal{O}_{X}\left(\left(r_{e}-1\right) D\right)$ splits as an $\mathcal{O}_{X}$-module, but $F^{e}: \mathcal{O}_{X} \rightarrow F_{*}^{e} \mathcal{O}_{X}\left(r_{e} D\right)$ does not split. Then $r_{e} \geq\left\lceil c p^{e}\right\rceil$ for all $e \in \mathbb{N}$. In particular, $(X, c D)$ is F-pure, and

\footnotetext{
${ }^{1}$ This rephrasal goes through since we are assuming that $R$ is regular, so that $1 \in R$ is a test element; see [HY].
} 


$$
\operatorname{fpt}(X, D)=\lim _{e \rightarrow \infty} \frac{r_{e}}{p^{e}}=\max \left\{t \in \mathbb{R}_{\geq 0} \mid(X, t D) \text { is F-pure }\right\} .
$$

Proof. Fix any $e \in \mathbb{N}$ and set $c^{\prime}=\left\lceil c p^{e}-1\right\rceil / p^{e}$. Then, since $c^{\prime}<c=\operatorname{fpt}(X, D)$, the pair $\left(X, c^{\prime} D\right)$ is strongly F-regular by Remark 2.5. Hence there exists $e^{\prime} \geq e$ such that the map $F^{e^{\prime}}: \mathcal{O}_{X} \rightarrow F_{*}^{e^{\prime}} \mathcal{O}_{X}\left(\left\lceil c^{\prime} p^{e^{\prime}} D\right\rceil\right)=F_{*}^{e^{\prime}} \mathcal{O}_{X}\left(\left\lceil c p^{e}-1\right\rceil p^{e^{\prime}-e} D\right)$ splits. Since this map factors through $F_{*}^{e} \mathcal{O}_{X}\left(\left\lceil c p^{e}-1\right\rceil D\right)$, the map $F^{e}: \mathcal{O}_{X} \rightarrow F_{*}^{e} \mathcal{O}_{X}\left(\left\lceil c p^{e}-1\right\rceil D\right)$ also splits, so that $\left\lceil c p^{e}-1\right\rceil \leq r_{e}-1$. Thus $r_{e} \geq\left\lceil c p^{e}\right\rceil$.

Since $c>0$ and $\left\lceil c p^{e}-1\right\rceil$ is the largest integer that is less than $c p^{e}$, we have $\left\lfloor c\left(p^{e}-1\right)\right\rfloor \leq\left\lceil c p^{e}-1\right\rceil \leq r_{e}-1$. Hence the map $F^{e}: \mathcal{O}_{X} \rightarrow F_{*}^{e} \mathcal{O}_{X}\left(\left\lfloor c\left(p^{e}-1\right)\right\rfloor D\right)$ splits for all $e \in \mathbb{N}$, so that $(X, c D)$ is F-pure; see the proof of [HW2, Corollary 2.7].

Since $\left\{r_{e} / p^{e}\right\}_{e \in \mathbb{N}}$ is an increasing sequence bounded above by 1 , there is a limit $\lim _{e \rightarrow \infty} r_{e} / p^{e} \in \mathbb{R}$, which is at least $c=\operatorname{fpt}(X, D)$ by the above argument. It remains to prove that $c \geq \lim _{e \rightarrow \infty} r_{e} / p^{e}$. If $t<\lim _{e \rightarrow \infty} r_{e} / p^{e}$, then $\left\lfloor t\left(p^{e}-1\right)\right\rfloor \leq r_{e}-1$ for all $e \gg 0$, so that $(X, t D)$ is F-pure. We conclude that $c \geq \lim _{e \rightarrow \infty} r_{e} / p^{e}$.

Next we recall the definition of the generalized test ideal $\tau\left(\mathfrak{a}^{t}\right)$ of an ideal $\mathfrak{a}$ with exponent $t \geq 0$. We restrict ourselves, for simplicity, to the case where $R=$ $k\left[\left[x_{1}, \ldots, x_{d}\right]\right]$ is a $d$-dimensional complete regular local ring as in Notation 2.1. Then the injective envelope $E=E_{R}(R / \mathfrak{m}) \cong H_{\mathfrak{m}}^{d}(R)$ of the residue field of $R$ may be described as the module of inverse polynomials $E=\left(x_{1} \cdots x_{d}\right)^{-1} k\left[x_{1}^{-1}, \ldots, x_{d}^{-1}\right]$. The Frobenius map $F: E \rightarrow E$ acts on this module by $F(z)=z^{p}$.

Definition 2.7 ([HY]). Let $(R, \mathfrak{m})$ be a regular local ring of characteristic $p>0$, $\mathfrak{a} \subseteq R$ a non-zero ideal, and let $t$ be a nonnegative real number.

(1) The $\mathfrak{a}^{t}$-tight closure $0_{E}^{* \mathfrak{a}^{t}}$ of the zero submodule ${ }^{2}$ in $E=E_{R}(R / \mathfrak{m})$ is the submodule of $E$ consisting of all elements $z \in E$ such that for all large $q=p^{e}$,

$$
\mathfrak{a}^{\lceil t q\rceil} z^{q}=0 \text { in } E .
$$

(2) The generalized test ideal $\tau\left(\mathfrak{a}^{t}\right)$ of the ideal $\mathfrak{a}$ with exponent $t$ is defined by

$$
\tau\left(\mathfrak{a}^{t}\right)=\operatorname{Ann}_{R}\left(0_{E}^{* \mathfrak{a}^{t}}\right) .
$$

In this paper, we only consider the case where $\mathfrak{a}=f R$ is a non-zero principal ideal. In this case, we denote the ideal $\tau\left(\mathfrak{a}^{t}\right)$ by $\tau\left(f^{t}\right)$. Alternatively, we use the notation $\tau(X, t D)$, where $D=\operatorname{div}_{X}(f)$ is the divisor on $X=\operatorname{Spec} R$ defined by $f$.

Proposition $2.8([\mathrm{HY}],[\mathrm{HT}])$. With the above notation we have the following.

(1) The pair $(X, t D)$ is strongly F-regular if and only if $\tau\left(f^{t}\right)=R$.

(2) If $t \geq t^{\prime} \geq 0$, then $\tau\left(f^{t}\right) \subseteq \tau\left(f^{t^{\prime}}\right)$.

(3) If $n \in \mathbb{Z}_{\geq 0}$, then $\tau\left(f^{n+t}\right)=f^{n} \cdot \tau\left(f^{t}\right)$.

(4) For any $t \in \mathbb{R}_{\geq 0}$, there exists $\varepsilon>0$ such that $\tau\left(f^{t}\right)=\tau\left(f^{t+\varepsilon}\right)$.

Proof. The proofs of (1)-(3) are standard and left to the reader; see [HY]. To prove (4), fix any $t \geq 0$ and $u \in \tau\left(f^{t}\right)$. Then choosing $d \in R^{\circ}$ in [HT, Proposition 2.1] to be $f$, we see that there exists $e_{0} \in \mathbb{N}$ and $\phi_{e} \in \operatorname{Hom}_{R}\left(R^{1 / p^{e}}, R\right)$ for $e=0,1, \ldots, e_{0}$

\footnotetext{
${ }^{2}$ In general, we can define the $\mathfrak{a}^{t}$-tight closure of any submodule of a module. For example, the $\mathfrak{a}^{t}$-tight closure $I^{* \mathfrak{a}^{t}}$ of an ideal $I \subseteq R$ is defined to be the ideal consisting of all elements $z \in R$ such that $\mathfrak{a}^{[t q]} z^{q} \subseteq I_{M}^{[q]}$ for all large $q=p^{e}$. The generalized test ideal $\tau\left(\mathfrak{a}^{t}\right)$ is then represented as $\tau\left(\mathfrak{a}^{t}\right)=\bigcap_{I \subseteq R}\left(I: I^{* \mathfrak{a}^{t}}\right)$, where $I$ runs through all ideals of $R$.
} 
such that $u=\sum_{e=0}^{e_{0}} \phi_{e}\left(\left(d \cdot f^{\left\lceil t p^{e}\right\rceil}\right)^{1 / p^{e}}\right)=\sum_{e=0}^{e_{0}} \phi_{e}\left(f^{\left\lceil 1+t p^{e}\right\rceil / p^{e}}\right)$. Since $1 \in R$ is a test element in the sense of [HY, Theorem 6.4], each $\phi_{e}\left(f^{\left\lceil 1+t p^{e}\right\rceil / p^{e}}\right)$ lies in $\tau\left(f^{t+\left(1 / p^{e}\right)}\right)$ again by [HT, Proposition 2.1]. So, if we put $\varepsilon=1 / p^{e_{0}}$, then $u \in \tau\left(f^{t+\varepsilon}\right)$.

Definition 2.9. Let $(R, \mathfrak{m})$ be a regular local ring of characteristic $p>0$ and let $f \in \mathfrak{m}$ be as in Notation 2.1. We say that a real number $c>0$ is an F-jumping exponent of the pair $(R, f)$ (or an F-jumping coefficient of the pair $(X, D)$ ), if $\tau\left(f^{t}\right) \supsetneq \tau\left(f^{c}\right)$ for all nonnegative $t<c$, or equivalently, if

$$
c=\inf \left\{t \in \mathbb{R}_{\geq 0} \mid \tau\left(f^{t}\right)=\tau\left(f^{c}\right)\right\} .
$$

This infimum is in fact a minimum because of Proposition 2.8 (4).

Remark 2.10. If $f \in \mathfrak{m}$, or equivalently, if $D=\operatorname{div}_{X}(f) \neq 0$, then there exist infinitely many F-jumping exponents of $(R, f)$, which coincide modulo $\mathbb{Z}$ with F-jumping exponents of $(R, f)$ in the interval $(0,1]$ by Proposition $2.8(3)$. Also by Proposition $2.8(1)$ and Remark 2.5, the smallest F-jumping exponent of the pair $(R, f)$ is nothing but the $\mathrm{F}$-pure threshold $\operatorname{fpt}(X, D)$. When the ring $R$ is regular, any $\mathrm{F}$-jumping exponent is an "F-threshold" defined as follows.

Definition 2.11. Let $(R, \mathfrak{m})$ be a regular local ring of characteristic $p>0$ and let $0 \neq f \in \mathfrak{m}$. Given any ideal $J$ containing a power of $f$, let $r_{e} \in \mathbb{N}$ be the integer such that $f^{r_{e}} \in J^{\left[p^{e}\right]}$ and $f^{r_{e}-1} \notin J^{\left[p_{e}\right]}$. Then let

$$
c_{J}(f)=\lim _{e \rightarrow \infty} \frac{r_{e}}{p^{e}} .
$$

In [MTW], this limit is called the $F$-threshold of $(R, f)$ with respect to $J$.

Proposition 2.12. Let $(R, \mathfrak{m})$ be a regular local ring of characteristic $p>0$ and $D=\operatorname{div}_{X}(f)$ the effective divisor on $X=\operatorname{Spec} R$ defined by $f \in \mathfrak{m}$ as in 2.1. Then

(1) $\operatorname{fpt}(X, D)=c_{\mathfrak{m}}(f)$.

(2) (Mustaţă-Takagi-Watanabe [MTW]) Let $c>0$ be an F-jumping exponent of the pair $(R, f)$ and let $J=\tau\left(f^{c}\right)=\tau(X, c D)$. Then $c=c_{J}(f)$.

Proof. (1) follows from Fedder's criterion ([F], [HW2, Corollary 2.7]), which asserts that $(X, t D)$ is F-pure if and only if $f^{\lfloor t(q-1)\rfloor} \notin \mathfrak{m}^{[q]}$ for all $q=p^{e}$. (2) is immediate from [MTW, Proposition 2.7].

Remark 2.13. Proposition 2.12 tells us that an F-jumping exponent is an F-threshold with respect to a generalized test ideal. On the other hand, it is implicitly proven in [MTW, Proposition 2.7] that every F-threshold is in fact an F-jumping exponent. As we will see below, F-jumping exponents may be considered "F-analogues" of certain invariants defined in characteristic zero.

Discussion 2.14. Let us recall the definitions of $\log$ canonical (lc, for short) and Kawamata log terminal (klt, for short) pairs, and of multiplier ideals. We assume that $D$ is an effective divisor on $X=\operatorname{Spec} R$ for a regular local ring $(R, \mathfrak{m})$ with $\operatorname{dim} R=2$ or the base field $k$ is of characteristic zero, because we need the existence of a log resolution of the pair $(X, D)$, that is, a proper birational morphism $\mu: Y \rightarrow X$ from a nonsingular variety $Y$ such that the union of $\operatorname{Supp}\left(\mu^{*} D\right)$ and the exceptional set of $\mu$ is a simple normal crossing divisor on $Y$. Let $t$ be a nonnegative real number, $K_{X}$ and $K_{Y}$ denote the canonical divisors of $X$ and $Y$, respectively, and write $K_{Y}=$ 
$\mu^{*}\left(K_{X}+t D\right)+\sum_{i=1}^{s} a_{i} E_{i}$ with $a_{i} \in \mathbb{R}$ and $E_{i}$ a prime divisor on $Y$. Then the pair $(X, t D)$ is said to be $l c$ (resp. $k l t)$ if $a_{i} \geq-1$ (resp. $a_{i}>-1$ ) for all $i=1, \ldots, s$. Also, the multiplier ideal sheaf $\mathcal{J}(X, t D) \subseteq \mathcal{O}_{X}$ is defined by $\mathcal{J}(X, t D)=\mu_{*} \mathcal{O}_{Y}\left(K_{Y}-\right.$ $\left.\left\lfloor\mu^{*}\left(K_{X}+t D\right)\right\rfloor\right)=\mu_{*} \mathcal{O}_{Y}\left(\sum_{i=1}^{s}\left\lceil a_{i}\right\rceil E_{i}\right)$. (Since $X$ is an affine scheme, we identify $\mathcal{J}(X, t D)$ with the ideal $H^{0}\left(X, \mathcal{O}_{Y}\left(K_{Y}-\left\lfloor\mu^{*}\left(K_{X}+t D\right)\right\rfloor\right)\right) \subseteq R$.) The log canonical threshold of $(X, D)$ is defined to be

$$
\operatorname{lct}(X, D)=\sup \left\{t \in \mathbb{R}_{\geq 0} \mid(X, t D) \text { is log canonical }\right\} .
$$

Since $\mathcal{J}(X, t D) \subseteq \mathcal{J}\left(X, t^{\prime} D\right)$ for $t \geq t^{\prime}$, one may define jumping coefficients for the multiplier ideal $\mathcal{J}(X, t D)$ as in Definition 2.9. Namely, a real number $c>0$ is a jumping coefficient of the pair $(X, D)$ if $\mathcal{J}(X, t D) \supsetneq \mathcal{J}(X, c D)$ for all nonnegative $t<$ c. Obviously, the pair $(X, t D)$ is klt if and only if $\mathcal{J}(X, t D)=\mathcal{O}_{X}$; that is to say, if and only if $t<\operatorname{lct}(X, D)$. So the $\log$ canonical threshold $\operatorname{lct}(X, D)$ is the smallest jumping coefficient of $(X, D)$ (for multiplier ideals). Moreover, all the basic properties of $\mathrm{F}$ singularities, generalized test ideals and F-jumping exponents stated in Propositions 2.3 and 2.8 and Remarks 2.5 and 2.10 hold true also in this setup if we replace "F-pure", "strongly F-regular", "generalized test ideal $\tau(X, t D)$ ", "F-pure threshold" and "F-jumping exponent" by "lc", "klt", "multiplier ideal $\mathcal{J}(X, t D)$ ", "log canonical threshold" and "jumping coefficient (for multiplier ideals)", respectively. We refer the reader to $[\mathrm{K}],[\mathrm{L}]$ and $[\mathrm{ELSV}]$ for more detail.

To compare F-pure and $\log$ canonical thresholds, we assume $\operatorname{dim} X=2$, so that we can speak of the log canonical threshold lct $(X, D)$ in all characteristics. When the base field $k$ is of characteristic $p>0$, the F-purity of the pair $(X, t D)$ implies that $(X, t D)$ is lc [HW2, Theorem 3.3]. Hence we always have the inequality $\operatorname{fpt}(X, D) \leq$ $\operatorname{lct}(X, D)$. This inequality may fail to be an equality. But, roughly speaking, we have $\operatorname{fpt}(X, D) \rightarrow \operatorname{lct}(X, D)$ as $p \rightarrow \infty$. This can be made precise by considering reduction modulo $p$ of a pair defined over a field of characteristic zero. To illustrate this, let $(X, D)$ be a pair defined over $\mathbb{Q}$. Let $\left(X_{p}, D_{p}\right)$ be the reduction of $(X, D)$ defined over $\mathbb{F}_{p}$; this makes sense for large $p$. Then by [HY, Theorem 6.8], for any $t<\operatorname{lct}(X, D)$, there exists $p(t) \in \mathbb{N}$ such that $\tau\left(X_{p}, t D_{p}\right)=\mathcal{J}\left(X_{p}, t D_{p}\right)=\mathcal{O}_{X_{p}}$ for all primes $p \geq p(t)$. Therefore we have that

$$
\operatorname{lct}(X, D)=\lim _{p \rightarrow \infty} \operatorname{fpt}\left(X_{p}, D_{p}\right) .
$$

Example 2.15. Let $D=\operatorname{div}_{X}(f)$ be the divisor defined by $f \in R=\mathbb{F}_{p}[[x, y]]$ on $X=\operatorname{Spec} R$.

(1) Let $f=x^{3}-y^{2}$. Then we obtain a $\log$ resolution $\mu: Y \rightarrow X$ of $(X, D)$ by blowing up at a point three times. If we denote the exceptional curve of the $i$ th blowing-up by $E_{i}(i=1,2,3)$ and the strict transform of $D$ on $Y$ by $\tilde{D}$, respectively, then $K_{Y}=\mu^{*} K_{X}+E_{1}+2 E_{2}+4 E_{3}$ and $\mu^{*} D=\tilde{D}+2 E_{1}+3 E_{2}+6 E_{3}$, so that $K_{Y}=\mu^{*}\left(K_{X}+t D\right)-t \tilde{D}+(1-2 t) E_{1}+(2-3 t) E_{2}+(4-6 t) E_{3}$. Hence $(X, t D)$ is lc if and only if $t \leq \frac{5}{6}$, and thus $\operatorname{lct}(X, D)=\frac{5}{6}$. On the other hand, using Fedder's criterion (Proposition 2.12) we verify that $\operatorname{fpt}(X, D)=\frac{1}{2}$ if $p=2 ; \frac{2}{3}$ if $p=3 ; \frac{5}{6}$ if $p \equiv 1 \bmod 3$; and $\frac{5 p-1}{6 p}$ if $p \equiv 2 \bmod 3 .^{3}$ The jumping coefficients of $(X, D)$ are $\frac{5}{6}$ and $1 \bmod \mathbb{Z}$, and the F-jumping exponents are $\operatorname{fpt}(X, D)$ and $1 \bmod \mathbb{Z}$.

\footnotetext{
${ }^{3}$ There is an error in the description of [HW2, Example 2.9(3)].
} 
(2) Let $f=x y(x+y)(x-y)$. Then the jumping coefficients of $(X, D)$ are $\frac{1}{2}=$ $\operatorname{lct}(X, D), \frac{3}{4}$ and $1 \bmod \mathbb{Z}$. On the other hand, $\operatorname{fpt}(X, D)=\frac{1}{2}$ if $p=2$ or $p \equiv 1 \bmod 4$; and $\frac{p-1}{2 p}$ if $p \equiv 3 \bmod 4$. If $p=3$, then the $\mathrm{F}$-jumping exponents are $\frac{1}{3}=\operatorname{fpt}(X, D)$, $\frac{2}{3}$ and $1 \bmod \mathbb{Z}$. The ideals $\mathcal{J}(X, c D)$ and $\tau(X, c D)$ corresponding to the first, second and third (F-)jumping coefficients are $\mathfrak{m}=(x, y), \mathfrak{m}^{2}$ and $f R$, respectively.

Looking at the above examples, we are led to some questions: If the pair $(X, D)$ is defined over $\mathbb{Q}$, is the $\mathrm{F}$-pure threshold $\operatorname{fpt}\left(X_{p}, D_{p}\right)$ of the modulo $p$ reduction equal to the log canonical threshold $\operatorname{lct}(X, D)$ for infinitely many primes $p$ ? This seems a very difficult problem even in the case $\operatorname{dim} X=2$. Also, it is natural to ask the following question since the jumping coefficients (for multiplier ideals) of a pair $(X, D)$ form a discrete set of rational numbers.

Conjecture 2.16. Let $(R, \mathfrak{m})$ be a regular local ring of characteristic $p>0$ and suppose $f \in \mathfrak{m}$ as in Notation 2.1 .

(1) The F-jumping exponents of $(R, f)$ are rational numbers.

(2) The set of F-jumping exponents of $(R, f)$ is discrete. Equivalently, the set of ideals $\left\{\tau\left(f^{t}\right) \mid 0<t \leq 1\right\}$ is finite.

There is also another way to phrase Conjecture 2.16. We may assume $R=k\left[\left[x_{1}, \ldots, x_{d}\right]\right]$. The F-threshold $c_{J}(f)$ of $(R, f)$ with respect to an ideal $J$ that contains a power of $f$ is the infimum of the $a / q$ where $(a, q)$ runs over all pairs with $f^{a} \in J^{[q]}$. Proposition 2.12 shows that (1) of Conjecture 2.16 holds if the $c_{J}(f)$ are rational, while $(2)$ holds if the $c_{J}(f)$, where $J$ runs over the ideals containing a power of $f$, form a discrete set. Monsky's appendix shows that this is the case if $d=2$ and $k$ is finite. Indeed Remark A.3 and Theorems A.10 and A.12 of the appendix give:

Theorem 2.17. Let $(R, \mathfrak{m})$ be a two-dimensional regular local ring of characteristic $p>0$ with finite residue field $R / \mathfrak{m}$ and let $0 \neq f \in \mathfrak{m}$. Then the $c_{J}(f)$, where $J$ runs over the ideals containing a power of $f$, form a discrete set of rational numbers.

The discussion above now gives Conjecture 2.16 when $\operatorname{dim} R=2$ and $R / \mathfrak{m}$ is a finite field. Theorem 1.1 follows immediately.

\section{F-pure thresholds and the function $\varphi_{f}$ for degree $d$ forms}

In this section, $k$ will always denote an algebraically closed field of characteristic $p, f \in k[x, y]$ a homogeneous polynomial of degree $d$ and $D$ the divisor of zeros of $f$ on the projective line $\mathbb{P}^{1}$ over $k$. For a non-negative real number $t$, we say that the pair $\left(\mathbb{P}^{1}, t D\right)$ is $F$-split if the $e$-times iterated Frobenius map $F^{e}: \mathcal{O} \rightarrow F_{*}^{e} \mathcal{O}$ of $\mathbb{P}^{1}$, followed by the natural inclusion $F_{*}^{e} \mathcal{O} \hookrightarrow F_{*}^{e} \mathcal{O}(\lfloor(q-1) t D\rfloor)$,

$$
F^{e}: \mathcal{O} \rightarrow F_{*}^{e} \mathcal{O}(\lfloor(q-1) t D\rfloor),
$$

is injective for all $q=p^{e}$. The following lemma, which is easily verified by computing Čech cohomology, enables us to compute the F-pure threshold $c_{\mathfrak{m}}(f)$ in terms of the geometry of $\mathbb{P}^{1}$; cf. [F] and [HW2].

Lemma 3.1. The following conditions are equivalent.

(1) The pair $\left(k[[x, y]], f^{t}\right)$ is F-pure.

(2) The pair $\left(\mathbb{P}^{1}, t D\right)$ is F-split. 
(3) For all $q=p^{e}, f^{\lfloor t(q-1)\rfloor} \notin\left(x^{q}, y^{q}\right)$.

(4) The map

$$
F^{e}: H^{1}\left(\mathbb{P}^{1}, \mathcal{O}\left(K_{\mathbb{P}^{1}}\right)\right) \rightarrow H^{1}\left(\mathbb{P}^{1}, \mathcal{O}\left(q K_{\mathbb{P}^{1}}+\lfloor(q-1) t D\rfloor\right)\right)
$$

is injective for all $q=p^{e}$.

To determine the injectivity of the map in (4) of the above lemma, we employ the following

Lemma 3.2 ([HW1]). Let $A, B$ be integral divisors on a smooth projective curve $X$ with $0 \leq B \leq(p-1) B_{\text {red }}$, where $B_{\text {red }}=\sum_{P \in \operatorname{Supp}(B)} P$ is the reduced part of $B$. Then the map

$$
F: H^{1}\left(X, \mathcal{O}_{X}(A)\right) \rightarrow H^{1}\left(X, \mathcal{O}_{X}(p A+B)\right)
$$

is injective if $\operatorname{deg}\left(K_{X}+p A+B+B_{\mathrm{red}}\right)<0$.

Proof. The $\log$ de Rham complex $\Omega_{X}^{\bullet}\left(\log B_{\text {red }}\right)$ with $\log$ poles along $B_{\text {red }}$ induces a complex $F_{*} \Omega_{X}^{\bullet}\left(\log B_{\text {red }}\right)(B)=F_{*}\left(\Omega_{X}^{\bullet}\left(\log B_{\text {red }}\right) \otimes \mathcal{O}_{X}(B)\right)$ of $\mathcal{O}_{X}$-modules. Since $0 \leq B \leq(p-1) B_{\text {red }}$, this complex gives rise to an exact sequence

$$
0 \rightarrow \mathcal{O}_{X} \rightarrow F_{*} \mathcal{O}_{X}(B) \rightarrow \mathcal{B} \rightarrow 0,
$$

where $\mathcal{B} \subset F_{*} \Omega_{X}^{1}\left(\log B_{\text {red }}\right)(B)=F_{*} \mathcal{O}_{X}\left(K_{X}+B+B_{\text {red }}\right)$ is the first coboundary. Tensoring this sequence with $\mathcal{O}_{X}(A)$ and looking at the cohomology long exact sequence, we see that the injectivity of the Frobenius map follows from the vanishing of $H^{0}\left(X, \mathcal{O}_{X}(A) \otimes F_{*} \mathcal{O}_{X}\left(K_{X}+B+B_{\text {red }}\right)\right)=H^{0}\left(X, \mathcal{O}_{X}\left(K_{X}+p A+B+B_{\text {red }}\right)\right)$.

Proposition 3.3. Let $f \in k[x, y]$ be a form of degree $d$ with $r$ linear factors, and assume that no linear form appears in $f$ with exponent $>d / 2$. Then the pair $\left(\mathbb{P}^{1}, t D\right)$ is F-split for $0 \leq t \leq(2 p-r+2) / p d$. Hence

$$
c_{\mathfrak{m}}(f) \geq \frac{2 p-r+2}{p d} .
$$

Proof. Since F-purity is a closed condition with respect to the coefficient (Proposition $2.6)$, it suffices to show that the pair $\left(\mathbb{P}^{1}, t D\right)$ is $\mathrm{F}$-split for all $t<(2 p-r+2) / p d$. To prove this it is enough to show the injectivity of the map

$$
F^{e}: H^{1}\left(\mathbb{P}^{1}, \mathcal{O}\left(K_{\mathbb{P}^{1}}\right)\right) \rightarrow H^{1}\left(\mathbb{P}^{1}, \mathcal{O}\left(\left\lfloor p^{e}\left(K_{\mathbb{P}^{1}}+t D\right)\right\rfloor\right)\right)
$$

for all $e \in \mathbb{N}$. We note that $\lfloor t D\rfloor=0$ since all the coefficients of $D$ are at most $d / 2$ by our assumption. Hence the above map is a composition of maps

$$
F: H^{1}\left(\mathbb{P}^{1}, \mathcal{O}\left(q K_{\mathbb{P}^{1}}+\lfloor q t D\rfloor\right)\right) \rightarrow H^{1}\left(\mathbb{P}^{1}, \mathcal{O}\left(p q K_{\mathbb{P}^{1}}+\lfloor p q t D\rfloor\right)\right)
$$

with $q=p^{0}, \ldots, p^{e-1}$. To these we apply Lemma 3.2 with $A=q K_{\mathbb{P}^{1}}+\lfloor q t D\rfloor$ and $B=$ $\lfloor p q t D\rfloor-p\lfloor q t D\rfloor$. Since $B_{\text {red }}$ consists of $r$ points by our assumption and $\operatorname{deg}\lfloor p q t D\rfloor<$ $q(2 p-r+2)$ if $t<(2 p-r+2) / p d$,

$\operatorname{deg}\left(K_{\mathbb{P}^{1}}+p q K_{\mathbb{P}^{1}}+\lfloor p q t D\rfloor+B_{\text {red }}\right)<-2(1+p q)+q(2 p-r+2)+r=(q-1)(2-r) \leq 0$, giving injectivity.

Remark 3.4. In the situation of Proposition 3.3, it is easy to see that the log canonical threshold of the pair is $2 / d$. 
The above lower bound for F-pure thresholds was also obtained by Monsky by a different method [M3]. His ideas allow us to give for each $d$ (and $p$ ) a finite list containing the possible values of $c_{\mathfrak{m}}(f)$ for forms $f$ of degree $d$. For this purpose, let $q$ be a power of $p, a$ an integer with $0 \leq a \leq q$, and consider the following exact sequence on $\mathbb{P}^{1}$,

$$
0 \rightarrow \mathcal{O}\left(-c_{1}\right) \oplus \mathcal{O}\left(-c_{2}\right) \rightarrow \mathcal{O}(-p)^{2} \oplus \mathcal{O}(-a d) \stackrel{\left(x^{p}, y^{p}, f^{a}\right)}{\longrightarrow} \mathcal{O} \rightarrow 0
$$

Monsky calls the absolute value $\left|c_{1}-c_{2}\right|$ the "syzygy gap."

Definition 3.5. With notation as above, define functions $\varphi=\varphi_{f}$ and $\delta=\delta_{f}$ on $[0,1]$ to be the continuous prolongations of the functions on $[0,1] \cap \mathbb{Z}[1 / p]$ whose values at $a / q$, where $a \in \mathbb{Z} \cap[0, q]$ and $q$ is a power of $p$, are as follows.

(1) $\varphi(a / q)=q^{-2} \operatorname{dim}_{k} k[x, y] /\left(x^{q}, y^{q}, f^{a}\right)$.

(2) $\delta(a / q)=\left|c_{1}-c_{2}\right| / q$, where $\left|c_{1}-c_{2}\right|$ is the syzygy gap for the sequence $(\dagger)$.

Note that the F-pure threshold is $c_{\mathfrak{m}}(f)=\inf \left\{t \in[0,1] \mid \varphi_{f}(t)=1\right\}$.

Lemma 3.6 ([M3]).

$$
\varphi(t)=d \cdot t-\frac{d^{2}}{4} t^{2}+\frac{\delta(t)^{2}}{4} .
$$

The first non-trivial case is when $f$ is a separable quartic form, i.e., $d=r=4$. In this case we have

Corollary 3.7. If $d=r=4$, then $c_{\mathfrak{m}}(f)=1 / 2$ or $(p-1) / 2 p$.

Proof. By Lemma 3.6, $\varphi(t)=4 t-4 t^{2}+\delta(t)^{2} / 4$. This in particular implies that $\delta((p-1) / 2 p)^{2} \leq 4 / p^{2}$, so that $\delta((p-1) / 2 p)=0$ or $2 / p$.

In the first case, $\varphi((p-1) / 2 p)<1$, so that $f^{(p-1) / 2} \notin\left(x^{p}, y^{p}\right)$. But this implies that $f^{(q-1) / 2} \notin\left(x^{q}, y^{q}\right)$ for all $q$, since for any $q \neq 1, f^{(q-1) / 2} \notin\left(x^{q}, y^{q}\right) \Leftrightarrow\left(z^{2}-f\right)^{q-1} \notin$ $\left(x^{q}, y^{q}, z^{q}\right) \Leftrightarrow k[[x, y, z]] /\left(z^{2}-f\right)$ is F-pure. Hence $c_{\mathfrak{m}}(f)=1 / 2$ in this case.

In the second case, one has $\delta((p-1) / 2 p)=1$, so that $c_{\mathfrak{m}}(f) \leq(p-1) / 2 p$. Proposition 3.3 shows that equality holds.

Remark 3.8. If $X$ is the elliptic curve obtained as the double cover of $\mathbb{P}^{1}$ ramified over the four zeros of $f$, then $X$ is ordinary if $c_{\mathfrak{m}}(f)=1 / 2$ and $X$ is supersingular if $c_{\mathfrak{m}}(f)=(p-1) / 2 p$.

The author was informed of the following result by Monsky.

Corollary 3.9 (Monsky). Suppose $d=r=5$.

$$
\begin{aligned}
& \text { If } p \equiv 1 \bmod 5, \text { then } c_{\mathfrak{m}}(f)=2 / 5 \text { or }(2 p-2) / 5 p ; \\
& \text { If } p \equiv 3 \bmod 5, \text { then } c_{\mathfrak{m}}(f)=(2 p-1) / 5 p ; \\
& \text { If } p \equiv 2 \bmod 5, \text { then } c_{\mathfrak{m}}(f)=\left(2 p^{2}-3\right) / 5 p^{2} \text { or }\left(2 p^{3}-1\right) / 5 p^{3} ; \\
& \text { If } p \equiv 4 \bmod 5, \text { then } c_{\mathfrak{m}}(f)=2 / 5,(2 p-3) / 5 p \text { or }\left(2 p^{2}-2\right) / 5 p^{2} \text {. }
\end{aligned}
$$

Our method will also prove this. For example, consider the case $p \equiv 4 \bmod 5$.

Since $d=5$, one has $\varphi(t)=5 t-25 t^{2} / 4+\delta(t)^{2} / 4$. It follows from $\varphi((2 p-3) / 5 p) \leq 1$ that $\delta((2 p-3) / 5 p) \leq 3 / p$. This implies that the syzygy gap for $q=p$ and $a=$ $(2 p-3) / 5 \in \mathbb{Z}$ is 1 or 3 , since $c_{1}-c_{2} \equiv c_{1}+c_{2}=2 p+5 a \equiv 1 \bmod 2$.

If $\delta((2 p-3) / 5 p)=3 / p$, then $\varphi((2 p-3) / 5 p)=1$, so that $c_{\mathfrak{m}}(f) \leq(2 p-3) / 5 p$. Proposition 3.3 then gives equality. 
Now assume that the syzygy gap is 1 . In this case, we prove

Claim 1. If $\delta((2 p-3) / 5 p)=1 / p$, then $c_{\mathfrak{m}}(f) \geq\left(2 p^{2}-2\right) / 5 p^{2}$.

It is enough to show the injectivity of the map

$$
F^{e}: H^{1}\left(\mathbb{P}^{1}, \mathcal{O}\left(K_{\mathbb{P}^{1}}\right)\right) \rightarrow H^{1}\left(\mathbb{P}^{1}, \mathcal{O}\left(\left\lfloor p^{e}\left(K_{\mathbb{P}^{1}}+t D\right)\right\rfloor\right)\right)
$$

for all $t<\left(2 p^{2}-2\right) / 5 p^{2}$ and all $e \in \mathbb{N}$. We write this map as a composition of maps

$$
F: H^{1}\left(\mathbb{P}^{1}, \mathcal{O}\left(q K_{\mathbb{P}^{1}}+\lfloor q t D\rfloor\right)\right) \rightarrow H^{1}\left(\mathbb{P}^{1}, \mathcal{O}\left(p q K_{\mathbb{P}^{1}}+\lfloor p q t D\rfloor\right)\right)
$$

with $q=p^{0}, \ldots, p^{e-1}$. Each of these maps with $q>1$ is injective. Indeed, if $q \geq p$, then $\lfloor p q t\rfloor<\left(2 p^{2}-2\right) q / 5 p \in \mathbb{Z}$, so that $\lfloor p q t\rfloor+1 \leq\left(2 p^{2}-2\right) q / 5 p$. Hence

$$
\operatorname{deg}\left(K_{\mathbb{P}^{1}}+p q K_{\mathbb{P}^{1}}+\lfloor p q t D\rfloor+D\right)=-2(1+p q)+5(\lfloor p q t\rfloor+1) \leq-2-\frac{2 q}{p}<0,
$$

and Lemma 3.2 applies. The injectivity for $q=p^{0}=1$ uses the syzygy gap assumption. Namely, it follows from $\delta((2 p-3) / 5 p)=1 / p$ that $\varphi(2 p-3) / 5 p)<1$. So $f^{(2 p-3) / 5}$ is not in $\left(x^{p}, y^{p}\right)$, and the map

$$
F: H^{1}\left(\mathbb{P}^{1}, \mathcal{O}\left(K_{\mathbb{P}^{1}}\right)\right) \rightarrow H^{1}\left(\mathbb{P}^{1}, \mathcal{O}\left(p K_{\mathbb{P}^{1}}+\frac{2 p-3}{5} D\right)\right)
$$

is injective. However, since $\lfloor p t\rfloor \leq\left\lfloor\left(2 p^{2}-2\right) / 5 p\right\rfloor=(2 p-3) / 5$, this map factors through $F: H^{1}\left(\mathbb{P}^{1}, \mathcal{O}\left(K_{\mathbb{P}^{1}}\right)\right) \rightarrow H^{1}\left(\mathbb{P}^{1}, \mathcal{O}\left(p K_{\mathbb{P}^{1}}+\lfloor p t\rfloor D\right)\right)$, yielding injectivity. This completes the proof of Claim 1.

We keep the assumption $\delta((2 p-3) / 5 p)=1 / p$ and consider next the syzygy gap for $q=p^{2}$ and $a=\left(2 p^{2}-2\right) / 5 \in 2 \mathbb{Z}$. It follows from $\varphi\left(\left(2 p^{2}-2\right) / 5 p^{2}\right) \leq 1$ that this syzygy gap is 0 or 2 , so that $\delta\left(\left(2 p^{2}-2\right) / 5 p^{2}\right)=0$ or $2 / p^{2}$.

In the latter case, we have $\varphi\left(\left(2 p^{2}-2\right) / 5 p^{2}\right)=1$, so that $c_{\mathfrak{m}}(f) \leq\left(2 p^{2}-2\right) / 5 p^{2}$. Claim 1 then shows that $c_{\mathfrak{m}}(f)=\left(2 p^{2}-2\right) / 5 p^{2}$. It remains to prove

Claim 2. If $\delta\left(\left(2 p^{2}-2\right) / 5 p^{2}\right)=0$, then $c_{\mathfrak{m}}(f)=2 / 5$.

Note that the assumption $\delta\left(\left(2 p^{2}-2\right) / 5 p^{2}\right)=0$ implies $f^{\left(2 p^{2}-2\right) / 5} \notin\left(x^{p^{2}}, y^{p^{2}}\right)$, so that the map

$$
F^{2}: H^{1}\left(\mathbb{P}^{1}, \mathcal{O}\left(K_{\mathbb{P}^{1}}\right)\right) \rightarrow H^{1}\left(\mathbb{P}^{1}, \mathcal{O}\left(p^{2} K_{\mathbb{P}^{1}}+\frac{2 p^{2}-2}{5} D\right)\right)
$$

is injective. This is the same as saying that the map $F^{2}: \mathcal{O} \rightarrow F_{*}^{2} \mathcal{O}\left(\frac{2 p^{2}-2}{5} D\right)$ splits as an $\mathcal{O}$-module homomorphism. Tensoring this map with $\mathcal{O}\left(\frac{2 p^{2}-2}{5} D\right)$ and pushing forward by $F^{2}$, we see that the map $F^{2}: F_{*}^{2} \mathcal{O}\left(\frac{2 p^{2}-2}{5} D\right) \rightarrow F_{*}^{4} \mathcal{O}\left(\frac{2 p^{4}-2}{5} D\right)$ splits as an $F_{*}^{2} \mathrm{O}$-module homomorphism. Composing this map with the previous one, we obtain the splitting of $F^{4}: \mathcal{O} \rightarrow F_{*}^{4} \mathcal{O}\left(\frac{2 p^{4}-2}{5} D\right)$. Iterating this, we see that the map $F^{2 e}: \mathcal{O} \rightarrow F_{*}^{2 e} \mathcal{O}\left(\frac{2}{5}\left(q^{2}-1\right) D\right)$ splits as an $\mathcal{O}$-module homomorphism for all $q=p^{e}$. Thus the pair $\left(\mathbb{P}^{1}, \frac{2}{5} D\right)$ is F-pure and $c_{\mathfrak{m}}(f)=2 / 5$.

This completes the proof of Corollary 3.9 in the case $p \equiv 4 \bmod 5$. The other cases are proved similarly.

We now consider $\varphi_{f}$ for large $p$. 
Theorem 3.10 (cf. [M3]). Let $f \in k[x, y]$ be a separable form of degree $d \geq 2$ with char $k=p>0$ and let the functions $\varphi=\varphi_{f}$ and $\delta=\delta_{f}$ be as in Definition 3.5. Then $\delta(a / p) \leq(d-2) / p$ for $0 \leq a \leq 2 p / d$. In particular, for fixed $d$,

$$
\varphi(t)=d \cdot t-\left(d^{2} / 4\right) t^{2}+O\left(1 / p^{2}\right) \text { on }[0,2 / d] .
$$

Proof. Consider the exact sequence $(\dagger)$ on $\mathbb{P}^{1}=\operatorname{Proj} k[x, y]$ used to defined $\delta(a / p)$. We have the following commutative diagram consisting of $(\dagger)$ together with $(\dagger)$ for $a=0$.

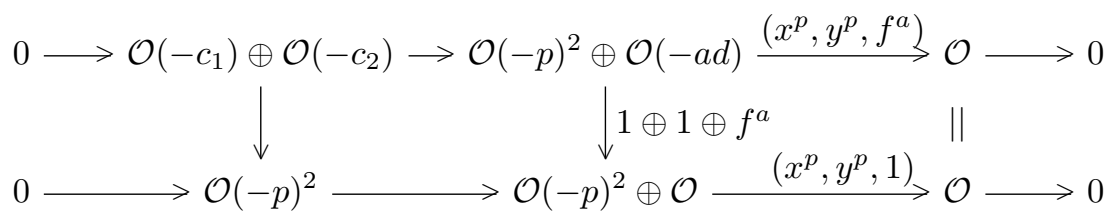

It follows that $c_{i} \geq p$ from the injectivity of the map $\mathcal{O}\left(-c_{1}\right) \oplus \mathcal{O}\left(-c_{2}\right) \rightarrow \mathcal{O}(-p)^{2}$ on the left. Assume that $c_{1} \leq c_{2}$. Then $c_{1} \leq p+a d / 2$ since $c_{1}+c_{2}=2 p+a d$. Our goal is to show that the syzygy gap $c_{2}-c_{1}$ is $\leq d-2$.

We represent the map $\mathcal{O}\left(-c_{1}\right) \oplus \mathcal{O}\left(-c_{2}\right) \rightarrow \mathcal{O}(-p)^{2} \oplus \mathcal{O}(-a d)$ in the exact sequence $(\dagger)$ by a $3 \times 2$-matrix and let $h_{1}, h_{2}, g \in k[x, y]$ be its first column entries; $g: \mathcal{O}\left(-c_{1}\right) \rightarrow$ $\mathcal{O}(-a d)$ is a form of degree $c_{1}-a d$, and $h_{1}, h_{2}$ are forms of the same degree $c_{1}-p$. Then the exactness of the sequence implies that $f^{a} g \in\left(x^{p}, y^{p}\right)$.

First, we consider the case $g=0$. In this case, $h_{1}, h_{2} \neq 0$ and $x^{p} h_{1}+y^{p} h_{2}=0$. Hence $y^{p}\left|h_{1}, x^{p}\right| h_{2}$ and $\operatorname{deg} h_{i} \geq p$, so that $2 p \leq c_{1} \leq p+a d / 2 \leq 2 p$. So the only possibility is that $a=2 p / d$. Then $f^{a} \in\left(x^{p}, y^{p}\right)$, and the sequence $(\dagger)$ is

$$
0 \rightarrow \mathcal{O}(-2 p)^{2} \rightarrow \mathcal{O}(-p)^{2} \oplus \mathcal{O}(-2 p) \rightarrow \mathcal{O} \rightarrow 0 .
$$

Thus the syzygy gap is $c_{2}-c_{1}=2 p-2 p=0$.

Now assume that $g \neq 0$, and let $E$ be the divisor of zeros of $g$ on $\mathbb{P}^{1}$. Suppose that $c_{2}-c_{1}>d-2$. Then $c_{1}<p+(a d-d+2) / 2$, so that

$$
\operatorname{deg}\left((p+1) K_{\mathbb{P}^{1}}+a D+E+(a D+E)_{\mathrm{red}}\right) \leq-2(p+1)+d(a+1)+2\left(c_{1}-a d\right)<0 .
$$

Hence by Proposition 3.2, the map

$$
F: H^{1}\left(\mathbb{P}^{1}, \mathcal{O}\left(K_{\mathbb{P}^{1}}\right)\right) \rightarrow H^{1}\left(\mathbb{P}^{1}, \mathcal{O}\left(p K_{\mathbb{P}^{1}}+a D+E\right)\right)
$$

is injective, contradicting the fact that $f^{a} g \in\left(x^{p}, y^{p}\right)$. Thus $c_{2}-c_{1} \leq d-2$, and this completes the proof.

\section{References}

[ELSV] L. Ein, R. Lazarsfeld, K.E. Smith, D. Varolin, Jumping coefficients of multiplier ideals, Duke Math. J. 123 (2004) 469-506.

[F] R. Fedder, F-purity and rational singularity, Trans. Amer. Math. Soc. 278 (1983) 461-480.

[HT] N. Hara and S. Takagi, On a generalization of test ideals, Nagoya Math. J. 175 (2004) $59-74$.

[HW1] N. Hara and K-I. Watanabe., The injectivity of Frobenius acting on cohomology and local cohomology modules, Manuscripta Math. 90 (1996) 301-315.

[HW2] _ F-regular and F-pure rings vs. log terminal and log canonical singularities, J. Algebraic Geom. 11 (2002) 363-392.

[HY] N. Hara and K. Yoshida, A generalization of tight closure and multiplier ideals, Trans. Amer. Math. Soc. 355 (2003) 3143-3174. 
[K] J. Kollár, Singularities of pairs, in “Algebraic Geometry—Santa Cruz 1995," pp. 221-287, Proc. Symp. Pure Math. 62, American Mathematical Society, Providence, 1997.

[L] R. Lazarsfeld, Positivity in Algebraic Geometry II, Ergebnisse der Math. Vol. 49, SpringerVerlag, 2004.

[M] P. Monsky, The Hilbert-Kunz function, Math. Ann. 263 (1983) 43-49.

[M2] - On the Hilbert-Kunz function of $z^{D}-p_{4}(x, y)$, J. Algebra 291 (2005) 350-372.

[M3] - Mason's theorem and syzygy gaps, J. Algebra, in press, available on-line.

[MT] P. Monsky and P. Teixeira, p-Fractals and power series-I. Some 2 variable results, Jour. Algebra 280 (2004) 505-536.

[MTW] M. Mustaţă, S. Takagi, and K-I. Watanabe, F-threshold and Bernstein-Sato polynomials, European Congress of Mathematics, 341-364, Eur. Math. Soc., Zürich, 2005.

[TW] S. Takagi, and K-I. Watanabe, On F-pure thresholds, J. Algebra 282 (2004) 278-297.

\section{Appendix A}

Paul Monsky

$k$ is a field of characteristic $p>0, q$ will always denote a power of $p,(A, \mathfrak{m})$ is the local ring $k\left[\left[x_{1}, \ldots, x_{s}\right]\right]$ and $f \neq 0$ is a fixed element of $\mathfrak{m}$. $J$ is an ideal of $A$ that contains a power of $f$. $\operatorname{deg} J$ is the colength of $J$; it may be $\infty$.

Definition A.1. $c_{J}=c_{J}(f)$ is the infimum of all $\frac{a}{q}$, where $(a, q)$ runs over the pairs with $f^{a} \in J^{[q]}$.

Conjecture A.2 (Hara). Let $J$ range over all ideals containing a power of $f$. Then the set of all $c_{J}$ has finite intersection with $[0,1]$.

Remark A.3. It's easily verified that $c_{J[p]}=p \cdot c_{J}$ and that $c_{[J: f]}=c_{J}-1$, provided $c_{J} \geq 1$. So if Conjecture A.2 holds, the set of all $c_{J}$ is a discrete subset of $[0, \infty)$. Furthermore, if $u$ is in this set so are all the $p^{n} u-\left\lfloor p^{n} u\right\rfloor$. So some two of these numbers are equal, and $u \in \mathbb{Q}$.

Remark A.4. In the attached article Hara shows that certain rationality conjectures for "F-pure thresholds" and "F-jumping coefficients" follow from Conjecture A.2.

Suppose from now on that $s=2$ and that the field $k$ is finite. We shall use $p$ fractal techniques from $[\mathrm{MT}]$ to prove Conjecture A.2. Write $(f)=\prod_{i=1}^{r}\left(f_{i}^{e_{i}}\right)$ with $f_{i}$ pairwise prime irreducibles. Since $s=2, J=H I$ with $\operatorname{deg} I<\infty$. We shall assume $c_{J}<1$. Then $f^{q-1} \in(H)^{q}$ for large $q$; it follows that $(H)=\left(\prod f_{i}^{b_{i}}\right)$ with $b_{i}<e_{i}$. Since there are only finitely many possibilities for $b_{1}, \ldots, b_{r}$, it suffices to prove Conjecture A. 2 where $J$ ranges over the ideals $\left(\prod f_{i}^{b_{i}}\right) I$, with $b_{i}<e_{i}$ fixed, and $\operatorname{deg} I<\infty$. From now on we fix $b_{1}, \ldots, b_{r}$.

Definition A.5. If $g$ is a continuous non-decreasing function on $[0,1], c(g)$, the critical point of $g$, is the infimum of all $t$ with $g(t)=g(1)$.

Lemma A.6. Let $g_{1}, \ldots, g_{n}$ be continuous non-decreasing functions on $[0,1]$. If $c_{i}=c\left(g_{i}\right)$ are distinct and $>0$, then $g_{1}, \ldots, g_{n}$ are linearly independent over $\mathbb{R}$.

Proof. We may assume $0<c_{1}<\cdots<c_{n}$. Suppose $\sum a_{i} g_{i}=0$. Restricting this relation to $\left[c_{n-1}, c_{n}\right]$, where each of $g_{1}, \ldots, g_{n-1}$ is constant but $g_{n}$ is not, we find that $a_{n}=0$, and an induction completes the proof. 
Definition A.7 (See further [MT]). Suppose $\operatorname{deg} I<\infty$.

(a) $\Phi_{I}:[0, \infty)^{r} \rightarrow[0, \infty)$ is the continuous function with

$$
\Phi_{I}\left(\frac{a_{1}}{q}, \ldots, \frac{a_{r}}{q}\right)=q^{-2} \operatorname{deg}\left(I^{[q]}, \prod f_{i}^{a_{i}}\right) .
$$

(b) $\Psi_{I}:[0,1]^{r} \rightarrow[0, \infty)$ is defined by:

(1) If any $e_{i} t_{i} \leq b_{i}$ then $\Psi_{I}\left(t_{1}, \ldots, t_{r}\right)=0$.

(2) Otherwise, $\Psi_{I}\left(t_{1}, \ldots, t_{r}\right)=\Phi_{I}\left(e_{1} t_{1}-b_{1}, \ldots, e_{r} t_{r}-b_{r}\right)$.

(c) $\psi_{I}(t)=\Psi_{I}(t, \ldots, t), 0 \leq t \leq 1$.

Note that $\psi_{I}$ is continuous and non-decreasing.

Lemma A.8. Suppose $J=\left(\prod f_{i}^{b_{i}}\right) I$, with $\operatorname{deg} I<\infty$. If $c_{J}<1$ then $c_{J}=c\left(\psi_{I}\right)$.

Proof. Since $c_{J}<1, f^{q} \in J^{[q]}$ for some $q, f \in J, \prod f_{i}^{e_{i}-b_{i}} \in I$, and $\psi_{I}(1)=$ $\Phi_{I}\left(e_{1}-b_{1}, \ldots, e_{r}-b_{r}\right)=\operatorname{deg} I$. Also

$$
\begin{aligned}
f^{a} \in J^{[q]} & \Leftrightarrow \prod f_{i}^{a e_{i}-q b_{i}} \in I^{[q]} \\
& \Leftrightarrow \Phi_{I}\left(\frac{a e_{1}-q b_{1}}{q}, \ldots, \frac{a e_{r}-q b_{r}}{q}\right)=\operatorname{deg} I \\
& \Leftrightarrow \psi_{I}\left(\frac{a}{q}\right)=\operatorname{deg} I,
\end{aligned}
$$

and the lemma follows.

Remark A.9. Suppose that the $\Psi_{I}$ of Definition A.7 span a finite dimensional real vector space. Then the same is true of the $\psi_{I}$, Lemmas A.6 and A.8 show that the $c_{J}$ that are $<1$ form a finite set, and Conjecture A.2 follows.

Theorem A.10. Suppose that $f$ is square-free, so that each $e_{i}=1$. Then Conjecture A.2 holds.

Proof. Now $b_{1}=\cdots=b_{r}=0$, and $\Psi_{I}$ is just the restriction of $\Phi_{I}$ to $[0,1]^{r}$. Since $k$ is finite we may invoke Proposition 3.5 of [MT], concluding that the space spanned by the $\Psi_{I}$ is finite-dimensional. (The key to the proof of Proposition 3.5 is Lemma 3.2 which says that there are only finitely many "ideal classes" in $k\left[\left[x_{1}, x_{2}\right]\right] / h$ when $k$ is finite and $h$ is square-free.)

We now treat arbitrary $f$, using a technique from $[\mathrm{MT}]$. An independent argument along similar lines has been given by Hara.

Let $V$ be the vector space of functions on $[0,1]^{r}$ spanned by the constant functions together with the restrictions to $[0,1]^{r}$ of the $\Phi_{I}, \operatorname{deg} I<\infty$. As we've noted, [MT] shows that $\operatorname{dim} V<\infty$. For each integer vector $d=\left(d_{1}, \ldots, d_{r}\right)$ with $0 \leq d_{i}<e_{i}$, let $X(d)$ consist of all $\left(t_{1}, \ldots, t_{r}\right)$ in $[0,1]^{r}$ with each $e_{i} t_{i}$ in $\left[d_{i}, 1+d_{i}\right]$.

Lemma A.11 (See further $[\mathrm{MT}]$ ). The restriction of $\Psi_{I}$ to any $X(d)$ is $\left(t_{1}, \ldots, t_{r}\right) \mapsto G\left(e_{1} t_{1}-d_{1}, \ldots, e_{r} t_{r}-d_{r}\right)$ for some $G$ in $V$.

Since $V$ is finite dimensional and there are only finitely many $X(d)$, this shows that the $\Psi_{I}$ span a finite dimensional space and we conclude:

Theorem A.12. Theorem A.10 is true without restriction on $f$. 
It remains to prove Lemma A.11. Suppose first that $d_{j}<b_{j}$ for some $j$. Then for each $\left(t_{1}, \ldots, t_{r}\right)$ in $X(d), e_{j} t_{j}-b_{j} \leq 0$. So $\Psi_{I}$ vanishes on $X(d)$ and we take $G=0$. Assume then that $d_{i} \geq b_{i}, 1 \leq i \leq r$. Set $g=\prod f_{i}^{d_{i}-b_{i}}$ and $I_{0}=[I: g]$. Suppose that $\left(\frac{a_{1}}{q}, \ldots, \frac{a_{r}}{q}\right)$ is in $X(d)$. Then $\Psi_{I}\left(\frac{a_{1}}{q}, \ldots, \frac{a_{r}}{q}\right)=q^{-2} \operatorname{deg}\left(I^{[q]}, \prod f_{i}^{a_{i} e_{i}-q b_{i}}\right)$. Let $s_{i}=$ $a_{i} e_{i}-q d_{i}$, so that $0 \leq s_{i} \leq q$. Then $\Psi_{I}\left(\frac{a_{1}}{q}, \ldots, \frac{a_{r}}{q}\right)=q^{-2} \operatorname{deg}\left(I^{[q]}, g^{q} \prod f_{i}^{s_{i}}\right)=\operatorname{deg} I-$ $q^{-2} \operatorname{deg}\left(I^{[q]}: g^{q} \prod f_{i}^{s_{i}}\right)$. This is evidently equal to $\operatorname{deg} I-\operatorname{deg} I_{0}+q^{-2} \operatorname{deg}\left(I_{0}^{[q]}, \prod f_{i}^{s_{i}}\right)$ $=\operatorname{deg} I-\operatorname{deg} I_{0}+\Phi_{I_{0}}\left(\frac{s_{1}}{q}, \ldots, \frac{s_{r}}{q}\right)$. Since $\frac{s_{i}}{q}=e_{i}\left(\frac{a_{i}}{q}\right)-d_{i}$, we conclude that Lemma A.11 holds if we take $G$ to be the sum of the constant function $\operatorname{deg} I-\operatorname{deg} I_{0}$ and the restriction to $[0,1]^{r}$ of $\Phi_{I_{0}}$; this completes the proof.

Remark A.13. The above approach to Conjecture A.2 relies heavily on Proposition 3.5 of [MT]. When $s \geq 3$ there is good reason to believe that this Proposition does not always hold. So our attack on Hara's conjectures may have limited use in higher dimensions.

Mathematical Institute, Tohoku University, Sendai 980-8578, Japan

E-mail address: hara@math.tohoku.ac.jp

Brandeis University, Waltham, MA 02454-9110

E-mail address: monsky@brandeis.edu 